\title{
Beneficial effects of multicomponent functional foods in patients with metabolic syndrome
}

\author{
Simona Dragan ${ }^{1}$, Georgiana Damian ${ }^{1 *}$, Dana Velimirovici ${ }^{1}$, Maria Rada ${ }^{1}$, Delia Berceanu ${ }^{1}$, \\ Mircea Iurciuc ${ }^{1}$, Marius Badalica-Petrescu ${ }^{1}$, Mihaela Valcovici ${ }^{1}$, Carmen Socaciu ${ }^{2}$, \\ Ruxandra Christodorescu ${ }^{1}$
}

1 University of Medicine and Pharmacy Victor Babes, 2 Eftimie Murgu Sq, 300041 Timisoara, Romania; E-mails: simona.dragan@umft.ro ;danavelimirovici@yahoo.com;radamariam@gmail.com berceanu.delia@umft.ro;mirceaiurciuc@gmail.com;marius_badalica@yahoo.com; mihaeladanielacardio@gmail.com; ruxandra_christodorescu@yahoo.com

2 University of Agricultural Sciences and Veterinary Medicine, 3-5 Manastur Str, 400372 ClujNapoca, Romania, E-mail: carmen.socaciu@usamvcluj.ro

* Author to whom correspondence should be addressed; E-mail: georgiana.damian6@gmail.com

\begin{abstract}
The ANTIATERO-ALIM study was a randomized, parallel design nutritional trial testing functional food (FF) diets against the usual diet recommended in the metabolic syndrome. Functional meals included balsamic vinegar from apples and honey and grape juice enriched with polyphenols from seeds of red grapes. 300 patients with metabolic syndrome were randomized into 4 groups: gr.1: FF $+\Omega-3$ supplements, gr.2: FF, gr.3: $\Omega-3$ supplements, gr.4: control. IRHOMA insulin resistance, plasma lipids and oxidative stress were assessed at inclusion and at 6 months. After 6 months there was a very significant decrease of oxidative stress in group 3, followed by a significant decrease in groups 1 and 2 .
\end{abstract}

Keywords: metabolic syndrome, multicomponent functional foods, antioxidant bioactive ingredients, polyphenols

\section{Introduction}

Metabolic syndrome has long been recognized as the common denominator for a set of five features: abdominal obesity, high glucose and insulin levels, low HDL, high triglycerides and high blood pressure [1]. Oxidative stress is thought to be linked to cardiovascular diseases, since oxidation of LDL in the vascular endothelium is a precursor to atherosclerotic plaque formation [2]. It is well known that atherosclerosis is associated with an increased production of reactive oxygen species and a reduction in antioxidant defences. Antioxidant micronutrients have been shown to significantly contribute to reduction or prevention of diabetic complications and atherosclerosis [3]. The Dietary 
Approaches to Stop Hypertension (DASH) trials featured controlled nutrient intake and repeated measurements, allowing for longitudinal analyses of biomarkers and traditional CVD risk factors [4]. The participants following the DASH diet had increases in serum antioxidants and oxygen radical absorbance capacity (ORAC) levels, and reduced breath ethane levels. Similarly in the DASH Sodium Trial, those on the DASH diet had increases in serum antioxidant and ORAC levels, lowered $\mathrm{F}_{2}$ isoprostane levels and increased antibodies to oxidized LDL [5].

The importance of efficient interventions to reduce obesity-linked risk has increased in recent decades because of the epidemic proportions of this condition. This seems to be particularly important for early intervention in high-risk individuals with metabolic syndrome, the cornerstone for cardiovascular diseases (CVD) and diabetes. Population-based strategies for healthy eating are being developed, among them recommendations for consumption of functional and novel foods with proven effects on biomarkers [6].

Functional foods typically contain small quantities of extra-nutritional constituents named bioactive compounds. These compounds vary widely in chemical structure and function and are grouped accordingly. Phenolic compounds, including their subcategory, flavonoids, are present in all plants and have been studied extensively in cereals, legumes, nuts, olive oil, vegetables, fruits, tea, and red wine. Many phenolic compounds have antioxidant properties, and some epidemiologic studies have reported protective associations between flavonoids or other phenolics and CVD, while other studies have not found these associations [7][8][9]. Studies with beta-carotene have led to similar results [10].

Antioxidant research has also involved extensive animal dietary trials. These trials are used to document effects in functional food development. Resveratrol $\left(3,4^{\prime}, 5\right.$ trihydroxystilbene), a naturallyoccurring phenolic antioxidant, is synthesized by plants in response to attacks by fungi or bacteria; it is also known to possess an array of cardioprotective effects [11][12] . A recent study has shown resveratrol to protect against the metabolic changes associated with hypercaloric diets in mice with induced insulin resistance, hyperglycemia, and dyslipidemia [13]. In diabetic mice, resveratrol restored endothelial function in type 2 diabetes by inhibiting TNFalpha-induced activation of NAD(P)H oxidase and preserving eNOS phosphorylation, suggesting the potential for new treatment approaches to promote vascular health in metabolic diseases [14].

A recent study in a rat model with myocardial infarction indicates that salvianolic acid $\mathrm{B}$, a water-soluble polyphenolic antioxidant from sage (Salvia miltiorrhiza) has a protective potential against myocardial infarction injury and that protective effects may be due to its scavenging lipid peroxidation products, increasing endogenous antioxidant defence enzymes [15]. It has also been demonstrated that oral administration of salvianolic acid A can significantly improve glucose metabolism and inhibit oxidative injury as well as protect against impaired vascular responsiveness in streptozocin-induced diabetic rats [16].

Basil (Ocimum spp., Lamiaceae) contains a wide range of essential oils rich in phenolic compounds and a wide array of other natural products including polyphenols such as flavonoids and anthocyanins [17][18]. Aqueous extracts of basil have been shown to exert antithrombotic and 
antioxidant properties in rats [19]. Rosmarinic acid has been shown to prevent atherosclerosis by endothelial nitric oxide synthase activation [20].

The Coronary Artery Risk Development in Young Adults (CARDIA) study and its ancillary study, Young Adult Longitudinal Trends in Antioxidants (YALTA) observational studies utilised a young American cohort, aged 18-30 years, in a bi-ethnic, multi-centre, prospective, longitudinal design. Serum carotenoid concentrations including alfa- and betacarotene, zeaxanthin, lutein and betacryptoxanthin, blood markers of inflammation, oxidative stress and endothelial dysfunction - all potential risk factors for disease development - were analysed. Serum carotenoids were found to be inversely related to markers of inflammation, oxidative stress and endothelial dysfunction [21].

The aim of our study was to test multi-component functional foods with bioactive ingredients from the plants mentioned above, with proven antioxidant, anti-inflammatory and antiatherosclerotic properties in patients with metabolic syndrome.

\section{Materials and methods}

\subsection{Functional foods}

The functional foods tested in the study, created by the Biotechnology Laboratories SC Proplanta SA Cluj Napoca Romania, were: 1. balsamic vinegar from apples with honey, buckthorn berry (Hyppophae rhamnoides) juice and extracts from herbs with proven antioxidant activity - sage, basil and rosemary; 2 . the already marketed VITIS product, a hydro-alchoholic extract from skins and seeds of red grapes. The balsamic vinegar was obtained from common apple vinegar, to which 5\% buckthornberry juice and hydro-alchoholic extracts from sage, basil and rosemary in a proportion according to the standardized recipe have been added. The product contains antocyanins (pelargonidin, malvidin), phenolic acids (cafeic, cumaric, ferulic, galic), flavonoids (kaemferol and quercetin), free aminoacids, peptides, 15 oligoelements $(\mathrm{Mg}, \mathrm{K}, \mathrm{Zn}, \mathrm{Ca}, \mathrm{Fe})$ in bioavailable form and provitamin $\mathrm{A}$ (carotenoids). The concentration of polyphenols in $100 \mathrm{ml}$ of standardized product was $42 \mathrm{mg}$ expressed in galic acid equivalents, according to the Folin-Ciocalteu method and that of beta-carotene was 17 micrograms, determined by HPLC analysis with saponification of samples according to a method described elsewhere [22][23].

The VITIS product is a monomeric, dimeric and polymeric polyphenolic compound obtained by sparing extraction from seeds, ramifications and skins of grapes, all sub-products of winemaking. High performance liquid chromatography (HPLC) revealed 6 classes of phenolic compounds: phenolic acids, proantocyanidins, tanins, catechines, antocyanins, flavonoids. The Folin-Ciocalteu method for polyphenol quantitation revealed a concentration of $33 \mathrm{mg} / 100 \mathrm{ml}$ product, expressed in galic acid equivalents [22].

\subsection{Nutritional trial protocol}

300 patients with metabolic syndrome were randomized for intervention or non-intervention in a prospective, randomized, parallel design nutritional trial at the University of Medicine and Pharmacy Victor Babes Timisoara, Romania. All patients had at least 3 criteria for metabolic syndrome according 
to the National Cholesterol Education Program Adult Treatment Panel III guidelines in managing dyslipidemia. (ATP III) criteria [24]. The study was approved by the local Ethics Committee.

All participants were instructed to respect the usual diet for metabolic syndrome according to guidelines [24]. In prescribing the caloric intake we took into account the resting metabolic rate (Kcal), determined by bioimpedance on the InBody 720 body composition analyser, to which we added an extra $400 \mathrm{Kcal}$ for medium level of work and another 200-400 kcal daily if they were exercising regularly. The recommended diet was low fat (15\% of calories) with a polyunsaturated-to-saturated fatty acid ratio of 2.4 to 1 , moderate protein (20\% of calories), unrefined carbohydrates with low glycemic index (65\% of calories) and fiber (more than 40 grams daily). The consumption of $30 \mathrm{ml}$ olive oil to prepare salads was recommended to all participants. Compliance to the diet was monitored by an adapted version of the National Health and Nutrition Examination Survey (NHANES) Questionnaire [25].

Participants were randomized into 4 groups:

Group 1 = multi-component functional foods + omega3 supplements

Group 2 = functional foods alone

Group 3 = omega 3 supplements alone

Group $4=$ control

Participants in group 1 and 2 were instructed to add 2 tablespoons of balsamic vinegar and 1 tablespoon of VITIS to either a salad of raw, fresh vegetables or a vegetable soup daily, 5 days per week. Omega3 supplements were added in the form of gelatinous capsules of $500 \mathrm{mg}, 1$ daily, to participants in gr 1 and 3 in order to compare the effect of functional foods with that of omega 3 supplementation, alone or in combination, on oxidative stress and metabolic parameters using group 4 randomized to usual diet as control.

The effects of nutritional intervention in the 4 groups were assessed at inclusion and at 6 months by parameters of metabolic effect: HOMA IR score for insulin-resistance, lipid profile and parameters of oxidative stress: total radicalic activity of whole blood measured by FORMox, total liposoluble antioxidants from serum (ACL) and total hidrosoluble antioxidants from serum (ACW) measured by photochemoluminometry.

The level of circulating insulin was determined by ELISA, using the kit DSL-10-1600 ACTIVE Insulin, DRG Diagnostics GmbH, Marburg, Germany. Blood glucose was determined by the colorimetric method with glucooxidase-peroxidase, kit-Clinical Lab. Diagnosticum, Budapest; for serial cholesterol the enzymatic colorimetric method was used, kit-Clinical Lab. Diagnosticum, Budapest; serum triglycerides were determined by enzymatic colorimetric method using the kitsClinical Lab. Diagnosticum, Budapest. To determine HDL-cholesterol we used the Vitalab-Flex-PC analyser.

The radicalic activity of whole blood was measured using the FORT test based on the ability of colorimetric transition of metals such as iron to catalyze the transformation of hidroperoxide radicals in derivatives under the Fenton reaction. The test was performed on capillary blood collected from the tip of the finger on the FORMox analyzer, Callegari, Italy. Values> FORT 310 units were considered suggestive for increased oxidative stress. 
Total hydrosoluble antioxidants from serum (ACW) and total liposoluble antioxidants from serum (ACL) were measured by photochemoluminometry (Photochem, Analytik Jena, Germany), at inclusion and after 6 months of functional diet.

\section{Results}

The study sample consisted of 300 patients (158 males, 142 females). In these patients, the parameters of metabolic syndrome were measured i.e. waist circumference, triglycerides (TG), HDL cholesterol, systolic and diastolic blood pressure and fasting glucose. The general characteristics of all patients are represented in Table 1.

Table 1. Characteristics of patients at inclusion $(n=300)$

\begin{tabular}{|l|l|l|}
\hline & Variable & \multicolumn{1}{|c|}{$\mathbf{n = 3 0 0}$} \\
\hline 1 & Age (yrs) & $54.25 \pm 11.08$ \\
\hline 2 & Sex & $158 \mathrm{M}, 142 \mathrm{~F}$ \\
\hline 3 & Glycemia ( mg/dl) & $101.66 \pm 33.9$ \\
\hline 4 & Systolic BP (mmHg) & $145.83 \pm 20.25$ \\
\hline 5 & Diastolic BP (mmHg) & $88.91 \pm 10.85$ \\
\hline 6 & TC (mg/dl) & $219.52 \pm 51.37$ \\
\hline 7 & HDL (mg/dl) & $49.57 \pm 12.26$ \\
\hline 8 & TG (mg/dl) & $185.97 \pm 86.58$ \\
\hline 9 & Waist Circumference (cm) & $\mathrm{M}: 114.78 \pm 3.21 \quad \mathrm{~F}: 110.17 \pm 2.57$ \\
\hline 10 & Body Mass Index(kg/m $\left.\mathbf{m}^{2}\right)$ & $\mathrm{M}: 35.65 \pm 9.96 \mathrm{~F}: 35.13 \pm 0.78$ \\
\hline 11 & FORM ox( uFORT) & $327.89 \pm 85.32$ \\
\hline 12 & IR HOMA & $4.82 \pm 3.32$ \\
\hline
\end{tabular}

Main characteristics were compared within the four groups at inclusion and after six months. 280 patients were compliant to the end of the study, there were 20 drop-outs.

Evolution of metabolic syndrome parameters in patients on functional foods and omega 3 supplements (group $1, \mathrm{n}=70$ ) are presented in Table 2. Total cholesterol registered a significant decrease after 6 months from $217.92 \pm 50.93$ to $208.12 \pm 40.71 \mathrm{mg} / \mathrm{dl}(\mathrm{p}=0.01)$, TG decreased significantly from $192.61 \pm 97.08$ to $160.58 \pm 125.51 \mathrm{mg} / \mathrm{dl}(\mathrm{p}=0.01)$ and HDL increased from $45.57 \pm 18.34$ to $52.59 \pm 11.81 \mathrm{mg} / \mathrm{dl}(\mathrm{p}=0.003)$. Group 1 showed a significant decrease in IRHOMA after 6 months from $5.34 \pm 3.93$ to $4.38 \pm 2.38$ ( $\mathrm{p}=0.01)$ and a very significant decrease in Form-ox after 6 months from $315.11 \pm 85.94$ to $275.41 \pm 84.34$ UFORT $(\mathrm{p}=0.001)$.

Table 2. Evolution of metabolic syndrome in patients on FF and $\Omega 3$ supplements (Group 1, $\mathrm{n}=70$ )

\begin{tabular}{|l|l|l|l|}
\hline Variables & \multicolumn{1}{|c|}{ Inclusion } & 6 months & p value \\
\hline Total cholesterol (mg/dl) & $217.92 \pm 50.93$ & $208.12 \pm 40.71$ & 0.01 \\
\hline
\end{tabular}




\begin{tabular}{|l|l|l|l|}
\hline Triglycerides(mg/dl) & $192.61 \pm 97.08$ & $160.58 \pm 125.51$ & 0.01 \\
\hline HDL (mg/dl) & $45.57 \pm 18.34$ & $52.59 \pm 11.81$ & 0.003 \\
\hline IRHOMA & $5.34 \pm 3.93$ & $4.38 \pm 2.38$ & 0.01 \\
\hline Form-ox (UFORT) & $315.11 \pm 85.94$ & $275.41 \pm 84.34$ & 0.001 \\
\hline BMI & $34.53 \pm 2.86$ & $32.86 \pm 1.97$ & 0.02 \\
\hline
\end{tabular}

Evolution of metabolic syndrome parameters in patients on functional foods only (group 2, $\mathrm{n}=65$ ) are presented in Table 3. TC showed a significant decrease after 6 months from $216.1 \pm 42.41$ to $205.74 \pm 34.59 \mathrm{mg} / \mathrm{dl}(\mathrm{p}=0.02)$, of TG from $177.34 \pm 92.20$ to $163.05 \pm 112.48 \mathrm{mg} / \mathrm{dl}(\mathrm{p}=0.02)$ and a very significant increase in HDL from $45.45 \pm 13.62$ to $51.97 \pm 9.41 \mathrm{mg} / \mathrm{dl}(\mathrm{p}=0.001)$. Group 2 showed a significant decrease in IRHOMA after 6 months from $4.72 \pm 3.73$ to $4.01 \pm 2.69(p=0.02)$ and also a significant decrease in Form-ox after 6 months from 317.93 \pm 91.91 to 290.04 \pm 82.86 UFORT $(\mathrm{p}=$ $0.01)$.

Table 3. Evolution of metabolic syndrome in patients on FF (Group 2, $n=65$ )

\begin{tabular}{|l|l|l|l|}
\hline Variables & \multicolumn{1}{|c|}{ Inclusion } & \multicolumn{1}{c|}{$\mathbf{6}$ months } & p value \\
\hline Total cholesterol (mg/dl) & $216.1 \pm 42.41$ & $205.74 \pm 34.59$ & 0.02 \\
\hline Triglycerides(mg/dl) & $177.34 \pm 92.20$ & $163.05 \pm 112.48$ & 0.02 \\
\hline HDL (mg/d) & $45.45 \pm 13.62$ & $51.97 \pm 9.41$ & 0.001 \\
\hline IRHOMA & $4.72 \pm 3.73$ & $4.01 \pm 2.69$ & 0.02 \\
\hline Form-ox(UFORT) & $317.93 \pm 91.91$ & $290.04 \pm 82.86$ & 0.01 \\
\hline BMI & $34.16 \pm 3.23$ & $32.19 \pm 2.78$ & $<0.01$ \\
\hline
\end{tabular}

Evolution of metabolic syndrome parameters in patients on omega 3 supplements (group 3, $n=75$ ) are presented in Table 4. TC showed a significant decrease after 6 months from $228.5 \pm 49.72$ to $214.56 \pm 39.61 \mathrm{mg} / \mathrm{dl}(\mathrm{p}=0.03)$, a very significant decrease in TG from $203.06 \pm 103.63$ to $166.45 \pm 80.45$ $\mathrm{mg} / \mathrm{dl}(\mathrm{p}=0.001)$. Group 3 showed a very significant increase in HDL after 6 months from $44.03 \pm 12.85$ to $49.86 \pm 8.88 \mathrm{mg} / \mathrm{dl}(\mathrm{p}=0.004)$ and also a very significant decrease in Form-ox after 6 months from $327.36 \pm 73.73$ to $265.95 \pm 82.23$ UFORT $(\mathrm{p}=0.0001)$. Group 3 did not show any significant change in IRHOMA after 6 months $(p=N S)$.

Evolution of metabolic syndrome in the control group (group $4, \mathrm{n}=70$ ) showed an insignificant decrease in TC after 6 months from $215.68 \pm 48.10$ to $208.65 \pm 44.06 \mathrm{mg} / \mathrm{dl}(\mathrm{p}=\mathrm{NS})$ and in TG from $158.73 \pm 68.17$ to $152.79 \pm 43.30 \mathrm{mg} / \mathrm{dl}(\mathrm{p}=\mathrm{NS})$. HDL increased significantly after 6 months from $41.94 \pm 11.51$ to $44.47 \pm 13.77 \mathrm{mg} / \mathrm{dl}(\mathrm{p}=0.02)$. IRHOMA and Form-ox remained unchanged $(\mathrm{p}=\mathrm{NS})$, as shown in Table 5.

Table 4. Evolution of metabolic syndrome in patients on $\Omega 3$ supplements (Group 3, $n=75$ )

\begin{tabular}{|l|l|c|l|}
\hline Variables & \multicolumn{1}{|c|}{ Inclusion } & 6 months & p value \\
\hline Total cholesterol(mg/dl) & $228.5 \pm 49.72$ & $214.56 \pm 39.61$ & 0.03 \\
\hline Triglycerides(mg/dl) & $203.06 \pm 103.63$ & $166.45 \pm 80.45$ & 0.001 \\
\hline
\end{tabular}




\begin{tabular}{|l|l|l|l|}
\hline HDL(mg/dl) & $44.03 \pm 12.85$ & $49.86 \pm 8.88$ & 0.004 \\
\hline IRHOMA & $4.94 \pm 3.06$ & $4.65 \pm 2.20$ & NS \\
\hline Form-ox(UFORT) & $327.36 \pm 73.73$ & $265.95 \pm 82.23$ & 0.0001 \\
\hline BMI & $33.46 \pm 3.42$ & $32.87 \pm 1.54$ & 0.001 \\
\hline
\end{tabular}

Table 5. Evolution of metabolic syndrome in the control group (Group 4, $\mathrm{n}=70$ )

\begin{tabular}{|l|l|l|l|}
\hline Variables & \multicolumn{1}{|c|}{ Inclusion } & \multicolumn{1}{c|}{ 6 months } & p value \\
\hline Total cholesterol (mg/dl) & $215.68 \pm 48.10$ & $208.65 \pm 44.06$ & NS \\
\hline Triglycerides(mg/d) & $158.73 \pm 68.17$ & $152.79 \pm 43.30$ & NS \\
\hline HDL(mg/dl) & $41.94 \pm 11.51$ & $44.47 \pm 13.77$ & 0.02 \\
\hline IRHOMA & $4.57 \pm 2.90$ & $4.76 \pm 3.71$ & NS \\
\hline Form-ox(UFORT) & $327.79 \pm 86.62$ & $310.61 \pm 85.46$ & NS \\
\hline BMI & $33.64 \pm 2.93$ & $31.87 \pm 2.74$ & 0.05 \\
\hline
\end{tabular}

The decrease of BMI was significant in all groups, in the following order: gr. $3<$ gr. $2<$ gr. $1<$ gr. 4 . The comparative evolution of oxidative stress between groups is presented in Figure 1. Measurements were made at inclusion, at 3 months and at 6 months. There was a constant very significant decrease of oxidative stress in group 3 (Omega $3, \mathrm{p}<0.0001$ ), followed by a significant decrease in group 1 $(\mathrm{FF}+\mathrm{Omega} 3, \mathrm{p}<0.001)$ and group $2(\mathrm{FF}, \mathrm{p}<0.01)$. There was an insignificant decrease of oxidative stress in Group 4 (control, $\mathrm{p}=\mathrm{NS}$ ), placing the effect of interventions on oxidative stress in the following order Omega3 $>\mathrm{FF}+$ Omega3 $>\mathrm{FF}>\mathrm{Control}$ (Fig 1). 
Figure 1 : Comparison of Form-ox between the four groups

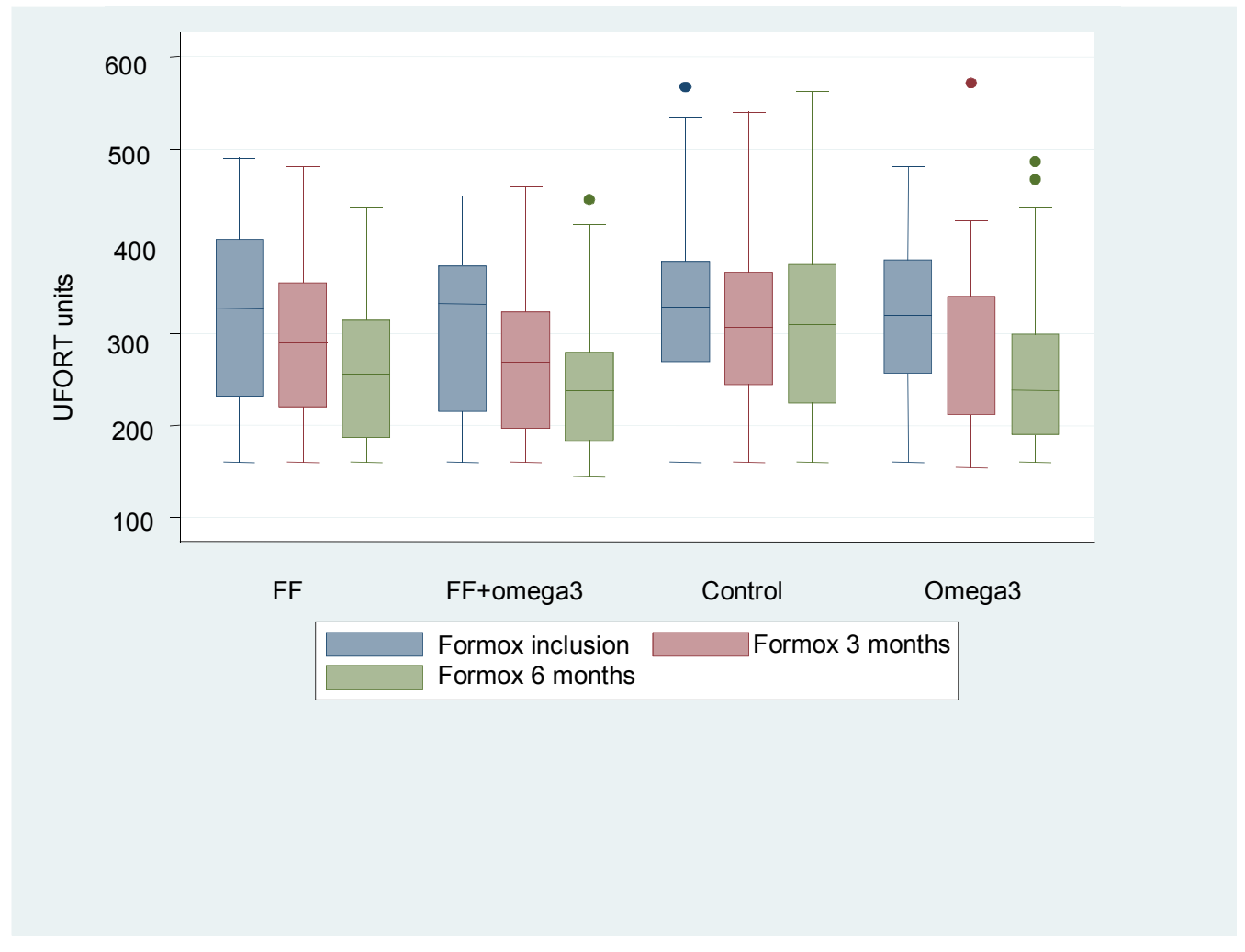

As shown in Figure 2, there was a significant increase of hydrosoluble antioxidants determined by photochemoluminometry in patients in group 1 on functional foods and omega 3 fatty acid supplements, from $339.49 \pm 41.49$ to $585.59 \pm 138.42$ micromoles/1 $(\mathrm{p}<0.05)$ and in group 2 on functional foods only from $354.53 \pm 32.25$ to $456.42 \pm 89.37$ micromoles $/ 1$ ( $\mathrm{p}=0.02$ ). 
Figure 2: Comparison of hydrosoluble antioxidants between the four groups

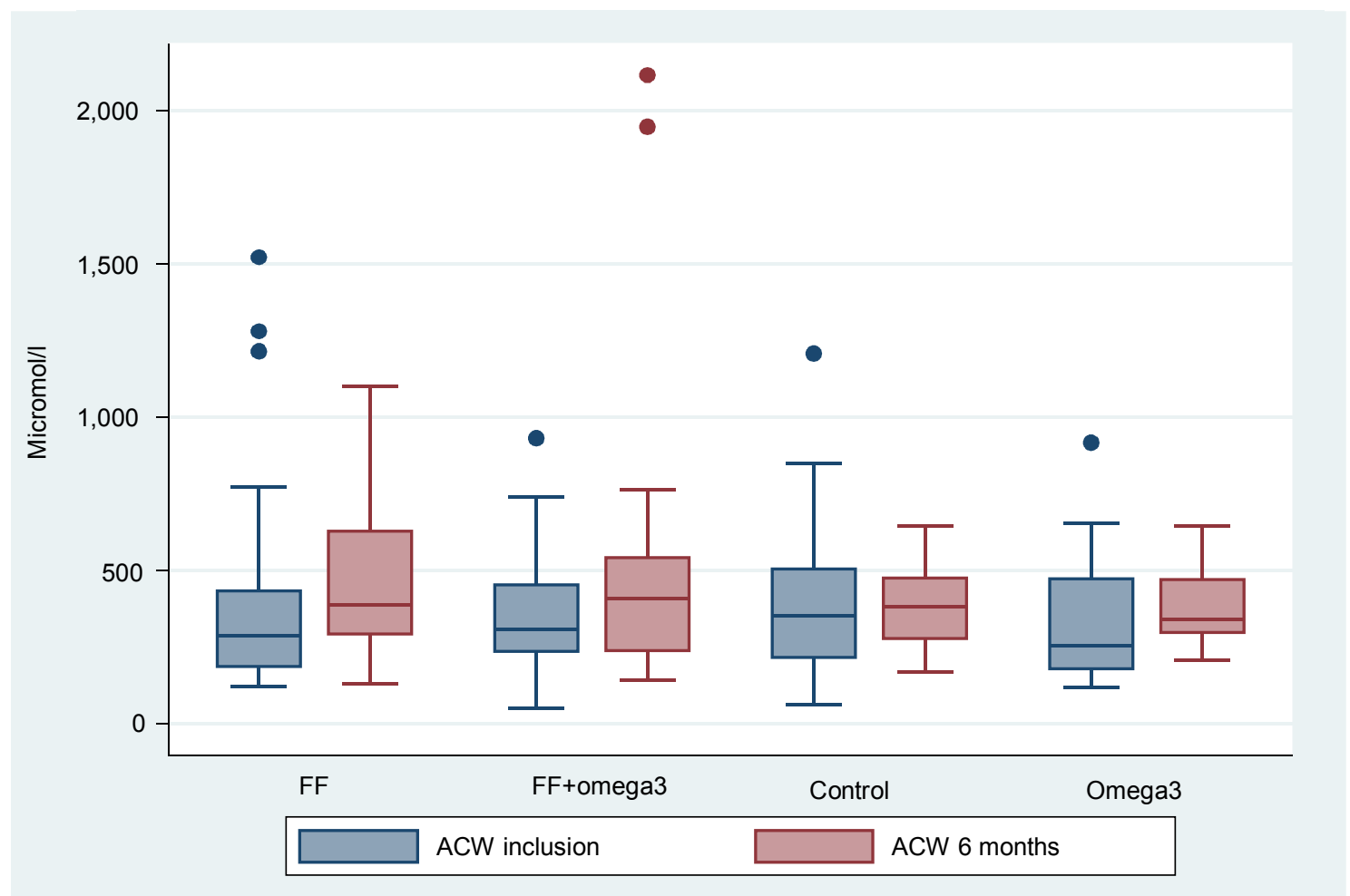

\section{Discussion}

Epidemiological studies have suggested positive associations between the consumption of phenolic-rich foods or beverages and the prevention of diseases [26]. These effects have been attributed to antioxidant components such as plant phenolics, including flavonoids and phenylpropanoids among others [27]. In our nutritional trial, designed to study the level of oxidative stress in response to a functional diet in patients with metabolic syndrome $(n=280)$, we demonstrated the functionality of the foods tested, balsamic vinegar from apples with honey, buckthorn berry juice (Hyppophae rhamnoides) and herbs with proven antioxidant activity - sage, basil and rosemary and the VITIS product, an extract from skins and seeds of red grapes.

The diet used was very simple, patients were asked to have one functional meal a day but only for 5 days (Monday - Friday) in a week in a randomized open trial. The 5/7 regimen was chosen to allow a better compliance and to imitate normal life conditions, where the same products cannot be eaten daily for six months. The products were easily available to the patients, some being already marketed products like the VITIS or the omega3 supplements. All other supplements were strictly forbidden during the trial. The decrease of oxidative stress was significantly enhanced by $\Omega 3$ supplements, that contributed also to significant decreases of TG levels. After 6 months of functional meals in the diet, group 1 showed the most significant decrease in the levels of glycemia, triglycerides, IRHOMA, insulin, Formox, compared to the diet recommended in the metabolic syndrome alone. So far, most of 
the cardio-protective effect of olive oil in the context of the Mediterranean diet has been attributed to its high MUFA content. In 2003, the Food and Drug Administration permitted a claim on olive oil labels concerning: "the benefits on the risk of coronary heart disease (CHD) of eating about 2 tablespoons (23 grams) of olive oil daily, due to the monounsaturated fat (MUFA) in olive oil" [28]. In our study, olive oil was also used in small amounts daily (max $30 \mathrm{ml}$ ) to prepare salads and the functional meals and its effect may also have contributed to our results, especially in the control group.

The oxidative stress induced by the metabolic syndrome is important in developing its complications, like diabetes mellitus, hypertension and cardiovascular diseases. Antioxidant micronutrients may thus significantly contribute to reduction or prevention of complications and atherosclerosis [29]. As antioxidants, polyphenols and carotenoids regulate the redox potential within the cells and activate signal transduction, important effects in disease prevention. Recent literature emphasizes potential therapeutic effects of micronutrients found in natural products, indicating positive applications for controlling the pathogenesis of chronic diseases. It is relevant that purified micronutrients isolated from natural products may be less effective than a combination seen in the natural product due to synergistic effects of interacting agents [30].

The use of herbal remedies has been on the rise worldwide. The safety of the plants used in the present study is evident by their use in folk medicine through time. Scientific evidence obtained so far is indicating antioxidant, properties of each of the plants. The main active ingredient in the grape skin extract VITIS, resveratrol, was recently reported to reduce endothelial oxidative stress by modulating the gene expression of superoxide dismutase 1 (SOD1), glutathione peroxidase 1 (GPx1) and NADPH oxidase subunit (Nox4), besides inhibition of LDL oxidation and suppression of platelet aggregation [31]. Rosemary (Rosmarinus officinalis L.) added to the balsamic vinegar is a very important medicinal and aromatic plant, which belongs to the Laminaceae family and has been cultivated for a long time. Rosmarinic acid has antibacterial, antioxidant and antiphlogistic effect [32]. SMND-309, a novel derivative of salvianolic acid $\mathrm{B}$, has been shown to significantly increased the activities of superoxide dismutase, catalase and glutathione peroxidase in a rat model of acute myocardial infarction [15]. These effects may account for decrease of oxidative stress in our study, added to the noted increase of ACW.

A recent review of current literature suggests that fruits and vegetables in combination have synergistic antioxidant effects leading to greater reduction in risk of chronic disease, specifically for cancer and heart disease, as established by the European Prospective Investigation into Cancer and Nutrition Study (EPIC) [33]. In our study, this synergistic antioxidant effect seems to have been achieved by the combination within the foods tested of the most important classes of antioxidants carotenoids and polyphenols, an effect suggested in Groups 1 and 2 by the constant and significant decrease of FORMox and by the increase of serum ACW.

There is a significant lack of understanding of the precise biological mechanisms of how plantbased bioactive food components impart health-promoting benefits. It is clear that bioactive food components act simultaneously at different or identical target sites. Bioactive food components have health-promoting roles at various stages of diseases that are associated with multiple progressive steps, from initiation to development. Isoflavones may reduce circulating oxidized low-density lipoproteins 
in the plasma, bind cholesterol in the intestinal tract, thereby reducing absorption of dietary cholesterol, enhance bile excretion reducing endogenous cholesterol levels, and modulate arterial elasticity improving blood vessel dilation and constriction response. [34]

Although there has been an increased emphasis on antioxidants and health in the literature, no 'gold-standard' method exists for assessing the health effects of antioxidants, and as such many methodologies have been developed. Results are therefore difficult to compare, and because of this are often conflicting. While more research is needed to define the 'best' study design for antioxidant studies, collaboration between the different scientific disciplines undertaking this research is needed to limit gaps in the literature and to ensure efficiency.

\section{Conclusion}

1. The functional products studied were well tolerated by all patients

2. The functional products studied decreased oxidative stress and contributed to an increase of water-soluble antioxidants in patients with metabolic syndrome

3. The decrease of oxidative stress was enhanced by omega-3 supplements, that also contributed to significant decreases of TG levels and increase of HDL

4. The functional products added to the diet brought lipid parameters closer to target and lowered IRHOMA, although a better control is needed

5. Long-term effects of functional diets seem promising for the control of metabolic syndrome parameters

Author Contributions: conceptualization- S.D. and R.C, data processing- M.B.P., M.I.; obtaining and characterization of functional foods-C.S., laboratory analysis- D.B., selection of patients and follow-up G.D., D.V., M.R., M.V. All authors contributed equally to this work. All authors approved the final version before submitting.

Funding: This research was funded by the Romanian Ministry of Research, program AGRAL.

Acknowledgments: nothing to declare

Conflicts of Interest: The authors declare no conflict of interest.

\section{Abbreviations}

FF Functional food

IRHOMA Homeostasis Model Assessment of Insulin Resistance

HDL High Density Lipoprotein 
LDL Low Density Lipoprotein

BMI Body Mass Index

DASH Dietary Approaches to Stop Hypertension

CVD CardioVascular Disease

ORAC Oxygen Radical Absorbance Capacity

TNF-alpha Tumor Necrosis Factor-alpha

NADPH Nicotinamide Adenine Dinucleotide Phosphate

eNOS endothelial Nitric Oxide Synthase

CARDIA Coronary Artery Risk Development in Young Adults

YALTA Young Adult Longitudinal Trends in Antioxidants

HPLC High Performance Liquid Chromatography

NHANES National Health and Nutrition Examination Survey

ACL total liposoluble antioxidants from serum

ACW total hidrosoluble antioxidants from serum

TC Total cholesterol

TG Triglyceride

CHD Coronary heart disease

MUFA Monounsaturated Fatty Acid

SOD1 Superoxide dismutase 1

GPx1 Glutathione peroxidase 1

EPIC European Prospective Investigation into Cancer and Nutrition Study

\section{References and Notes}

1. Scuteri A, Laurent S, Cucca F, Cockcroft J, Cunha G, Mañas LR, et al. HHS Public Access. 2016;22(4):486-91.

2. Ungvari Z, Tarantini S, Donato AJ, Galvan V, Csiszar A. Mechanisms of Vascular Aging. Circ 
Res [Internet]. 2018;123(7):849-67. Available from: https://www.ahajournals.org/doi/10.1161/CIRCRESAHA.118.311378

3. Alkhatib A, Tsang C, Tiss A, Bahorun T, Arefanian H, Barake R, et al. Functional foods and lifestyle approaches for diabetes prevention and management. Nutrients. 2017;9(12):1-18.

4. Chiu S, Bergeron N, Williams PT, Bray GA, Sutherland B, Krauss RM. Comparison of the DASH (Dietary Approaches to Stop Hypertension) diet and a higher-fat DASH diet on blood pressure and lipids and lipoproteins: A randomized controlled trial. Am J Clin Nutr. 2016;103(2):341-7.

5. Miller, ER 3rd, Erlinger, TP, Sacks, FM, Svetkey, LP, Charleston, J, Lin, PH, Appel L. A dietary pattern that lowers oxidative stress increases antibodies to oxidized LDL: results from a randomized controlled feeding study. Atherosclerosis. 2005;183:175-182.

6. Pérez-Martínez P, Mikhailidis DP, Athyros VG, Bullo M, Couture P, Covas MI, et al. Lifestyle recommendations for the prevention and management of metabolic syndrome: An international panel recommendation. Nutr Rev. 2017;75(5):307-26.

7. Olas B. Berry phenolic antioxidants - implications for human health? Front Pharmacol. 2018;9(MAR):1-14.

8. Barona J, Aristizabal JC, Blesso CN, Volek JS, Fernandez ML. Grape Polyphenols Reduce Blood Pressure and Increase Flow-Mediated Vasodilation in Men with Metabolic Syndrome. J Nutr [Internet]. 2012;142(9):1626-32. Available from: http://jn.nutrition.org/cgi/doi/10.3945/jn.112.162743

9. Barona J, Blesso CN, Andersen CJ, Park Y, Lee J, Fernandez ML. Grape consumption increases anti-inflammatory markers and upregulates peripheral nitric oxide synthase in the absence of dyslipidemias in men with metabolic syndrome. Nutrients. 2012;4(12):1945-57.

10. Eggersdorfer, M, Wyss A. Carotenoids in human nutrition and health. Arch Biochem Biophys. 2018;652:18-26.

11. Nawaz W, Zhou Z, Deng S, Ma X, Ma X, Li C, et al. Therapeutic versatility of resveratrol derivatives. Nutrients. 2017;9(11).

12. Riba A, Deres L, Sumegi B, Toth K, Szabados E, Halmosi R. Cardioprotective effect of resveratrol in a postinfarction heart failure model. Oxid Med Cell Longev. 2017;2017.

13. Sadruddin, S.; Arora R. Resveratrol: biologic and therapeutic implications. J Cardiometab Syndr. 2009;4:102-6.

14. Zhang H.; Zhang J.; Ungvari Z.; Zhang C. Resveratrol improves endothelial function: role of TNF \{alpha\} and vascular oxidative stress. Arter Thromb Vasc Biol. 2009;29:1164-71.

15. Yang J.; Zhang G.; Tian J.; Li C.; Jiang W.; Xing Y.; Zhu H.; Hou J.; Xu H.; Wu J. Cardioprotective effect of SMND-309, a novel derivate of salvianolic acid B on acute myocardial infarction in rats. Basic Clin Pharmacol Toxicol. 2009;([Epub ahead of print]).

16. Wang S.B.; Yang X.Y.; Tian S.; Yang H.G.; Du G.H. Effect of salvianolic acid A on vascular reactivity of streptozotocin-induced diabetic rats. Life Sci. 2009;85:499-504.

17. Lee,J.; Scagel CF. Chicoric acid levels in commercial basil (Ocimum basilicum) and Echinacea purpurea products. J Funct Foods. 2010;2:77-84.

18. Tarchoune I., Sgherrii C., Baâtour O., Izzo R., Lachaâ M., Navari-Izzo F., Ouerghi Z. Phenolic acids and total antioxidant activity in Ocimum basilicum L. grown under Na2SO4 medium. J Med Plants Res. 2012;6(48):5868-75.

19. Tohti I.; Tursun M.; Umar A.; Turdi S.; Imin H.; Moore N. Aqueous extracts of Ocimum basilicum L. (sweet basil) decrease platelet aggregation induced by ADP and thrombin in vitro and rats arterio-venous shunt thrombosis in vivo. Thromb Res. 2006;118:733-9.

20. Mueller M.; Lukas B.; Novak J.; Simoncini T.; Genazzani A.R.; Jungbauer A. Oregano: a source for peroxisome proliferator-activated receptor gamma antagonists. J Agric Food Chem. 2008;56:11621-30.

21. Park K.; Gross M.; Lee D-H.; Holvoet P.; Himes J.H; Shikany J.M.; Jacobs D.R. Oxidative 
stress and insulin resistance. The Coronary Artery Risk Development in Young Adults Study. Diabetes Care. 2009;32:1302-7.

22. Prior R.L.; Wu X.; Schaich K. Standardized methods for the determination of antioxidant capacity and phenolics in foods and dietary supplements. J Agric Food Chem. 2005;53:310113.

23. Chiosa V., Mandravel C., Kleinjans J.K.S. ME. Determination of beta-carotene concentration in orange and apple juice and in vitamin supplemented drinks. Analele Univ Buc - Chim. 2005;14:253-8.

24. Talbert RL. Role of the National Cholesterol Education Program Adult treatment panel III guidelines in managing dyslipidemia. Am J Heal Syst Pharm. 2003;60:3-8.

25. Block G. Foods contributing to energy intake in the US: data from NHANES III and NHANES 1999-2000. J Food Compos Anal. 2004;17:439-47.

26. Scalbert A.; Williamson A. Dietary intake and bioavailability of polyphenols. J Nutr. 2000;130:2073S-2085S.

27. Rice-Evans C.A.; Miller N.J.; Paganga G. Structure-antioxidant activity relationships of flavonoids and phenolic acids. Free Radic BiolMed. 1996;20:933-56.

28. F.D.A. Interim Procedures for Qualified Health Claims in the Labeling of Conventional Human Food and Human Dietary Supplements. $2003 . \quad$ p. (http://www.cfsan.fda.gov/ dms/nuttftoc.html).

29. Zhang, H.; Tsao R. Dietary polyphenols, oxidative stress and antioxidant and anti-inflammatory effects. Food Sci. 2016;8:33-42.

30. Soory M. Relevance of nutritional antioxidants in metabolic syndrome, ageing and cancer: potential for therapeutic targeting. Infect Disord Drug Targets. 2009;9:400-14.

31. Spanier G.; Xu H.; Xia N.; Tobias S.; Deng S.; Wojnowski L.; Forstermann U.; Li H. Resveratrol reduces endothelial oxidative stress by modulating the gene expression of superoxide dismutase 1 (SOD1), glutathione peroxidase 1 (GPx1) and NADPH oxidase subunit (Nox4). J Physiol Pharmacol. 2009; Suppl 4:111-6.

32. Stefanovits-Banyai E.; Tulok M.H.; Hegedus A.; Renner C.; Szollosi Varga I. Antioxidant properties of various rosemary clones. Acta Biol Szeged. 2003;47:111-3.

33. Gonzales CA. The European Prospective Investigation into Cancer and Nutrition (EPIC). Public Heal Nutr. 2006;9:124-6.

34. Zaheer, K.; Humayoun Akhtar M. An updated review of dietary isoflavones: Nutrition, processing, bioavailability and impacts on human health. J Crit Rev Food Sci Nutr. 2017;57:1280-93. 